\title{
Performance Analysis of Variable-Geometry Scramjet Inlets Using a Low-Order Model
}

\author{
Derek J. Dalle*, Sean M. Torrez ${ }^{\dagger}$, and James F. Driscoll ${ }^{\ddagger}$ \\ University of Michigan, Ann Arbor, MI 48109
}

\begin{abstract}
Scramjet vehicles, especially those used as part of an orbital launch system, must operate over a wide range of flight conditions. One component that has difficulty accommodating a range of Mach numbers is the inlet. In this article, only two-dimensional-type scramjet inlets are considered. Such an inlet with fixed geometry can be designed for a single Mach number (using approximately the shock-on-lip configuration) or a range of Mach numbers. However, the performance of the inlet tends to degrade as the size of the Mach number range increases. One method to improve this performance is to use a variable-geometry cowl. Three cowl motions are considered in this paper: moving the whole cowl up and down, moving the whole cowl forward and backward, and rotating the cowl lip. A low-order model designed for control-oriented applications is used to simulate wave interactions. The model is used to evaluate the benefits of each type of variable geometry, and an inlet designed for a wide range of Mach numbers is presented.
\end{abstract}

\section{Nomenclature}

$\begin{array}{lll}A & =\text { capture area per unit width } \\ H & =\text { height of inlet to cowl leading edge } \\ m & =\text { number of external ramps } \\ M & =\text { Mach number } \\ n & =\text { number of internal ramps } \\ N & =\text { total number of ramps } \\ p & =\text { pressure } \\ r & =\text { length of shock segment } \\ T & =\text { temperature } \\ u & =\text { input variable } \\ U & =\text { input variable upper limit } \\ x & =\text { horizontal coordinate } \\ z & =\text { vertical coordinate } \\ \alpha & =\text { angle of attack } \\ \beta & =\text { angle between shock and upstream flow } \\ \delta & =\text { deflection angle across a shock } \\ \theta & =\text { angle of flow from } x \text {-axis } \\ \lambda & =\text { dimensionless length }\end{array}$

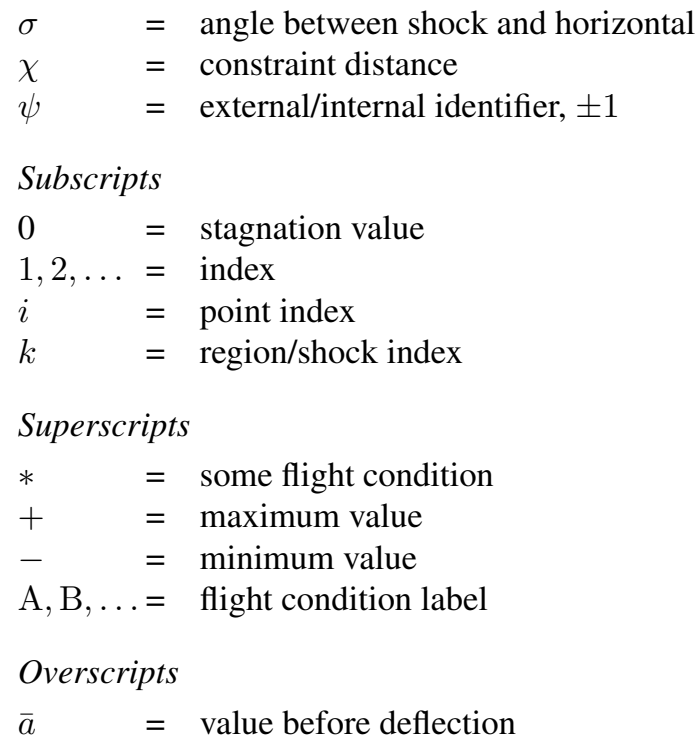

\section{Introduction}

Variable-geometry inlets have often been proposed or tested [1] as a way to improve performance of a vehicle that must perform over a wide range of Mach numbers. For hypersonic air-breathing vehicles, the inlet is responsible for providing much of the compression necessary for combustion, and, as a result, vehicle performance can be very sensitive to the inlet geometry. If the inlet is designed for a single condition, the performance may be poor at all other conditions [2,3]. At the same time, designing the inlet for a wider range of Mach numbers tends to decrease the performance at each individual condition [4]. With a variable-geometry inlet, the configuration changes with Mach number so that the performance does not fall off as greatly.

\footnotetext{
* Graduate Research Assistant, Department of Aerospace Engineering, AIAA Member, dal le@umich . edu

$\dagger$ Graduate Research Assistant, Department of Aerospace Engineering, AIAA Member, smtorrez@umich. edu

${ }^{\ddagger}$ Professor, Department of Aerospace Engineering, AIAA Fellow, james fd@umich . edu
} 
Actually building a variable-geometry hypersonic inlet presents numerous technical challenges. This paper does not address these physical aspects of the problem, but it aims to measure the potential benefit of allowing simple changes to the inlet geometry. We consider only three degrees of freedom, all of which pertain only to the inlet cowl: moving the entire cowl forward or backward, moving the entire cowl up or down, and rotating a flap at the front of the cowl. We show that these relatively simple parameters can improve inlet performance over a range of Mach numbers, and that one of these parameters, namely moving the cowl vertically, is more effective than the other two.

Hypersonic flight vehicles on which variable-geometry inlets were proposed include the National Aero-Space Plane (NASP) and the X-43C [5, 6]. The Ground Demonstrator Engine-Build 2 (GDE-2) included a rotating cowl flap that performed well in the Mach 4.5 to 6.5 range using realistic flight hardware including high-temperature seals [6]. A configurable inlet/isolator experiment was tested by Emami et al. [7] in which the cowl length and angle could be changed between test runs.

Some of the proposed variable-geometry inlets attempt to enforce the shock-on-lip constraint $[8,9]$ in which the bow shock is assumed to hit the front of the cowl, and an expansion at the shoulder is expected to cancel the reflected shock. Although meeting the shock-on-lip constraint has been shown to be neither plausible [10] nor desirable [4] they are simple to analyze and provide a baseline performance for comparison. Some of the trends obtained from the simplified shock-on-lip analysis are expected to apply to a more realistic design. For example, as the Mach number (and thus the altitude, assuming some reasonable trajectory) increase, more compression is needed, and one way to achieve this might be to move the entire cowl upward to achieve a higher compression ratio.

A more advanced analysis tool, called Supersonic Aerodynamic Analysis Using Riemann Interactions (SAMURI), has been developed [2] for rapid analysis of scramjet inlet flow, and this tool is used for this inlet investigation. SAMURI accounts for oblique shock waves, supersonic expansion fans, slip lines, and their interactions, and an example solution for a scramjet inlet is shown in Fig. 1. This tool provides a method to analyze off-design performance with accuracy approaching that of two-dimensional computational fluid dynamics (CFD), but SAMURI typically uses four seconds or less of computational time on a single $3.3 \mathrm{GHz}$ processor.

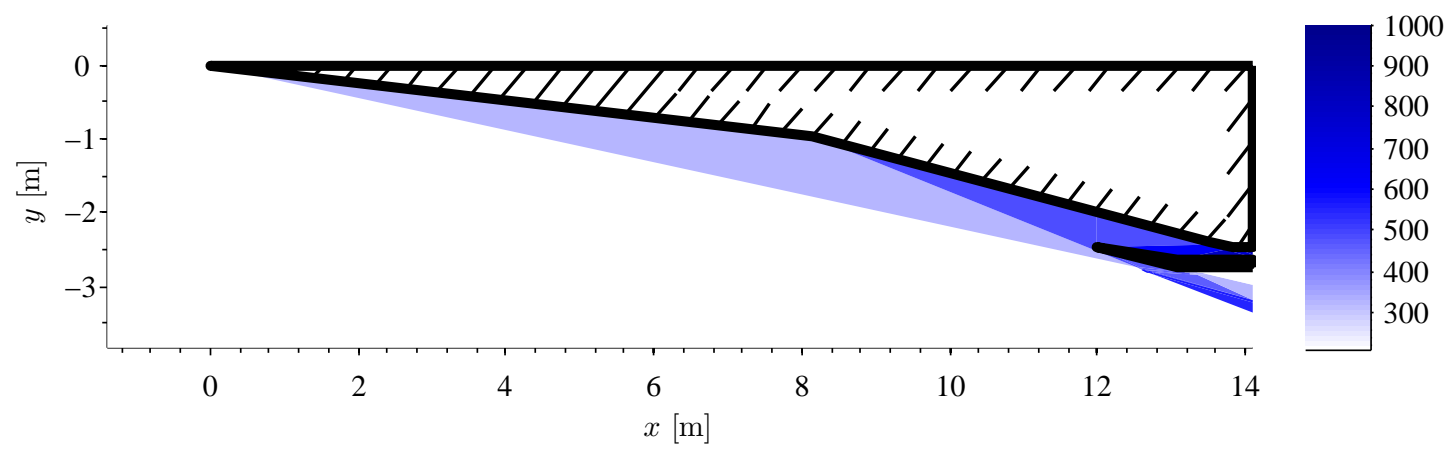

a) Full inlet.

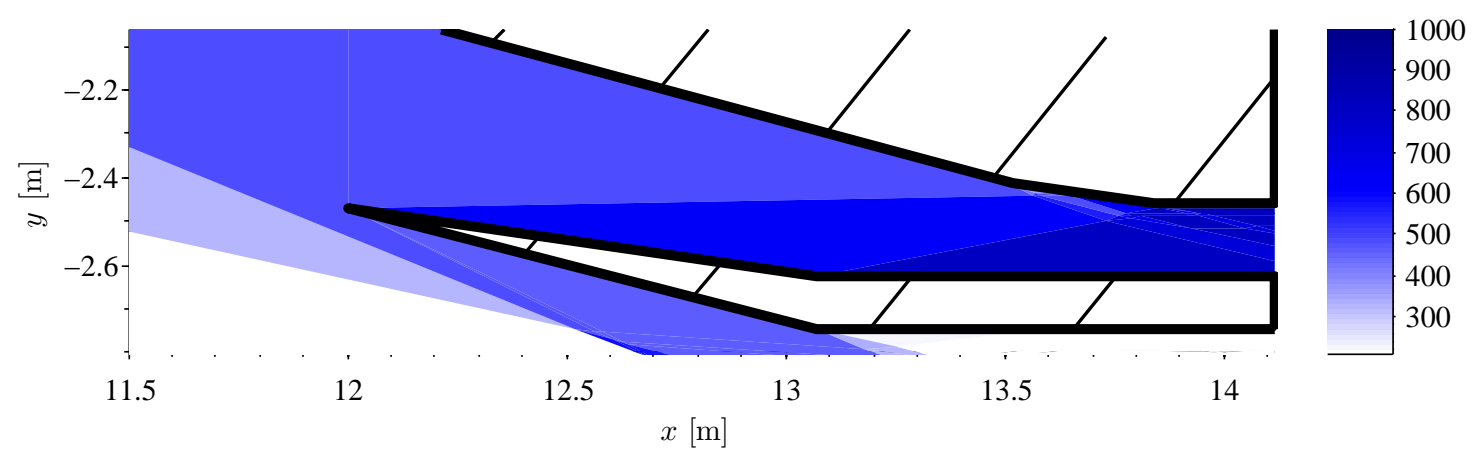

b) Cowl region.

Figure 1. Temperature contours for an example inlet solution flying at $M_{\infty}=8$ and $\alpha=0$. 


\section{Inlet Geometry and Definitions}

Figure 2 shows a sample inlet configuration with the relevant angles marked. It shows the inlet as essentially a sequence of oblique shocks. The first $m$ shocks deflect the freestream flow to a certain angle, and the last $n$ shocks turn the flow back to horizontal. In Fig. 2, $m=2$ and $n=2$. The resulting inlet consists of $N=m+n$ shocks and $2 m+n-2$ vertices, for which the numbering is shown in Fig. 2b.

During the initial design phase, we assume that the flow consists of only the shocks in Fig. 2, which are shown as gray lines. These shocks divide the flow into $N+1$ regions, and expansion fans, shock interactions, and wave reflections are ignored during the initial process. Once the inlet geometry has been designed, we apply the full SAMURI model to test the performance.

\section{A. Oblique Shock Theory}

During the initial inlet design phase, the flow analysis consists solely of oblique shocks. Each shock has three angles associated with it, and each flow region has another angle. The angle associated with each region is the flowpath angle, i.e. the angle between the flow direction and the $x$-axis.

We split the shocks into two families. The first $m$ shocks increase the flowpath angle, and the last $n$ shocks decrease the flowpath angle. We define a value $\psi_{k}$ for each shock such that $\psi_{k}=1$ for the first $m$ shocks and $\psi_{k}=-1$ for the last $n$ shocks. The deflection angle across the $k$ th shock is

$$
\delta_{k}=\left(\theta_{k+1}-\theta_{k}\right) \psi_{k}
$$

The angle between a given shock and the $x$-axis is $\sigma_{k}$, and the angle between the shock and the flow upstream of the shock is $\beta_{k}$. We define $\beta_{k}$ so that it is always positive. The relationship between these two angles is

$$
\sigma_{k}=\theta_{k}+\psi_{k} \beta_{k}
$$

Standard oblique shock theory [11] tells us that we can determine the conditions downstream of the shock as long as we know the upstream conditions and the deflection angle across the shock. The relationship between the deflection angle and shock angle is

$$
\tan \delta_{k}=\cot \beta_{k} \frac{M_{k}^{2} \sin ^{2} \beta_{k}}{\frac{\gamma+1}{2} M_{k}^{2}-M_{k}^{2} \sin ^{2} \beta_{k}+1}
$$

Then the downstream pressure is given by

$$
\frac{p_{k+1}}{p_{k}}=\frac{2 \gamma}{\gamma+1} M_{k}^{2} \sin ^{2} \beta_{k}-\frac{\gamma-1}{\gamma+1}
$$

and the downstream Mach number is given by

$$
M_{k+1}=\frac{1}{\sin \left(\beta_{k}-\delta_{k}\right)} \sqrt{\frac{1+\frac{\gamma-1}{2} M_{k}^{2} \sin ^{2} \beta_{k}}{\gamma M_{k}^{2} \sin ^{2} \beta_{k}-\frac{\gamma-1}{2}}}
$$

\section{B. Performance Metrics}

Some of the objectives of the inlet are to provide a flow into the combustor that has adequate static pressure and temperature, providing lift, and keeping both drag and stagnation pressure loss low. Finding the optimal inlet design for a given vehicle would require a simulation of the entire vehicle, but we can use a few dimensionless quantities that capture much of the performance.

The most obvious quantity is simply the compression ratio of the inlet, which in our simplified analysis is

$$
\frac{p_{N+1}}{p_{1}}=\prod_{k=1}^{N} \frac{p_{k+1}}{p_{k}}
$$

In reality the flow will not necessarily be uniform at the end of the inlet, and some average value for the static pressure in the flow would be more appropriate. As a guide to our initial design methodology, however, this equation will be reasonably accurate. A slightly more sophisticated parameter is called the pressure recovery factor. This compares the ratio of the final stagnation pressure to the freestream stagnation pressure, so the approximate formula is

$$
\begin{gathered}
\frac{p_{0, N+1}}{p_{0,1}}=\frac{p_{N+1}}{p_{1}}\left(\frac{1+\frac{\gamma-1}{2} M_{N+1}^{2}}{1+\frac{\gamma-1}{2} M_{1}^{2}}\right)^{\frac{\gamma}{\gamma-1}} \\
3 \text { of } 17
\end{gathered}
$$




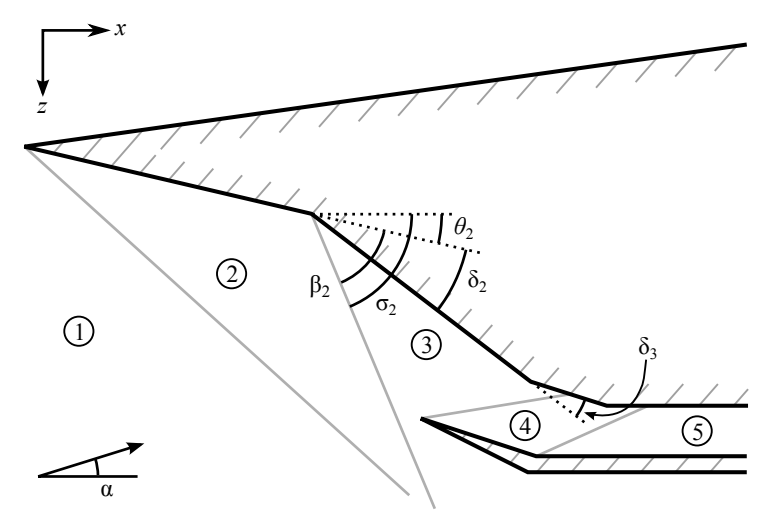

a) Angle definitions.

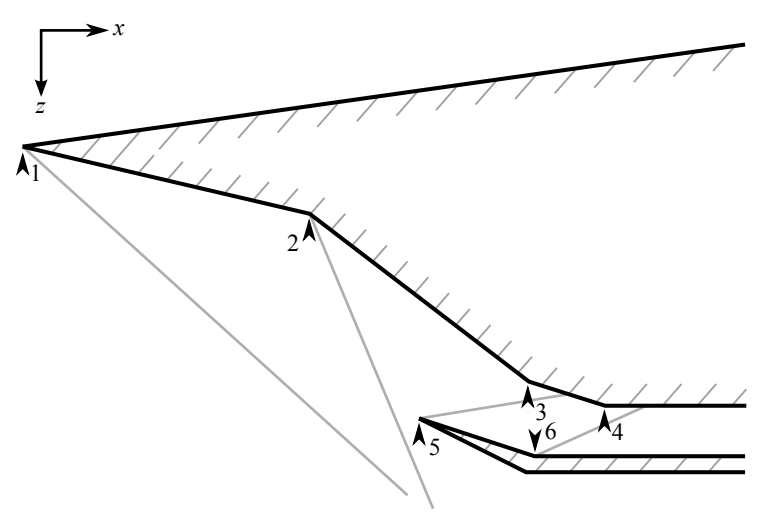

b) Vertex indices.

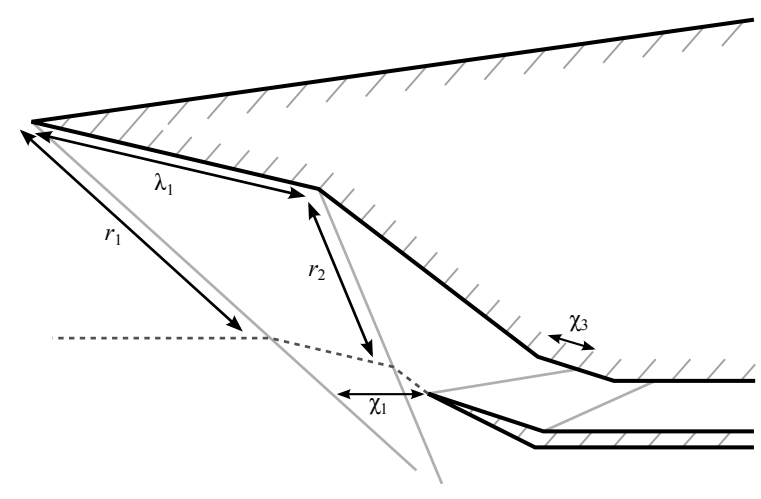

c) Length definitions.

Figure 2. Definitions for inlet design methodology.

The pressure recovery factor is a measure of how much entropy is generated by the inlet since the pressure recovery factor is always 1 for isentropic flow. It also is a prediction of drag because increasing the pressure recovery factor without changing the compression ratio means increasing the exit Mach number, $M_{N+1}$. The pressure recovery factor has also been used in other scramjet inlet optimization studies [12].

The final performance metric we consider in this discussion is the capture area. Figure $2 \mathrm{c}$ shows a dotted line that represents the last streamline that is captured by the inlet. The simplest way to find this line is to trace the streamline backwards from the cowl leading edge. The length of the $k$ th shock from the surface of the vehicle to the point that it intersects the last streamline is $r_{k}$. Then we define the normal area for each shock as

$$
A_{k}=r_{k} \sin \beta_{k}
$$

The capture area is just $A_{1}$. The idea of capture area is to get a measure of thrust; if more air is captured, and the pressure and temperature remain the same, we would expect the combustor to generate more thrust. A very closely related parameter is mass spillage. The maximum capture area is the height of inlet, so we define the spillage as $1-A_{1} / H$.

\section{Fixed-Geometry Definitions}

Because the oblique shock equations for a perfect gas can be written in non-dimensional form, we can model the flow knowing only the freestream Mach number and the sequence of deflection angles. To construct an inlet geometry, we also need the distances between vertices, $\lambda_{k}$. We put the first vertex, the leading edge of the inlet body, at the origin, and we write $\bar{x}_{N+1}=1$. This means that the inlet geometry is nondimensionalized by the distance in the $x$-direction from the leading edge of the inlet to the leading edge of the cowl. The $z$-coordinate of the cowl leading edge is not fixed, and we write $\bar{z}_{N+1}=H$ for convenience. The bar over the variables denotes the position for the initial 
geometry, i.e. with all of the variable-geometry inputs set equal to zero. We fix the $x$-coordinate of the cowl leading edge so that the geometry is also non-dimensional, but we still need the $z$-coordinate. This gives $2 N+n-2$ degrees of freedom, which are $\delta_{1}, \ldots, \delta_{N-1}, \lambda_{1}, \ldots, \lambda_{N+n-2}$, and $H$. We use the definition that $\theta_{N+1}=0$, but adding it as a degree of freedom is as simple as a change of coordinates.

With this framework for constructing the inlet, we turn our attention to selecting effective values of $\theta_{k}$ and $\lambda_{i}$. In [4], the authors discussed the need to account for the range of Mach number during the design process. The first constraint for good performance is that the external shocks (i.e. the first $m$ shocks) do not go into the internal flow. An external shock in the internal flow not only increases the heat load on the cowl leading edge but also interferes with the internal flow. So we write $\chi_{k}$ as the distance in the $-x$-direction from the cowl leading edge to the $k$ th external shock. In Fig. $2 c, \chi_{1}$ is marked. Preventing the shocks from going into the internal portion of the inlet is equivalent to requiring that $\chi_{k}$ is greater than or equal to zero for each external shock.

The desired behavior of the internal shocks is that shock $k$ hits the inlet body between vertex $k$ and vertex $k+1$. If the shock goes upstream of vertex $k$, it decreases the performance dramatically because the reflected shock will be very strong [13]. In addition, the boundary layer is likely to separate in that case. If the $k$ th shock goes downstream of vertex $k+1$, some of the flow must go through two expansions before it sees the shock, and the amount of interactions in the internal part of the inlet will be very high.

For shocks $n+1, \ldots, N$, we define $\chi_{k}$ to be the distance from vertex $k$ to the location at which the $k$ th shock hits the lower surface of the inlet body. In Fig. 2c, one of these distances, $\chi_{3}$, is marked. In order to keep each internal shock between the prescribed vertices, we use the constraint

$$
0 \leq \chi_{k} \leq \lambda_{k} \quad k=m+1, \ldots, N
$$

\section{Variable Geometry}

We consider three cowl motions, which are shown in Fig. 3, for the available variable geometry. The first allowable change to geometry is a movement of the entire cowl forward, as shown in Fig. 3a. Figure 3b shows the entire cowl deflected downward by a distance $u_{2}$, which is the second allowable motion. The third motion involves rotating the first section of the cowl flap by an angle $u_{3}$, as shown in Fig. 3c. The third geometry variation is different from the first two because it only affects one of the vertices. As a result, this is most useful when $n=2$. When $n=1$, there is no rotation available, and when $n>2$, some of the shocks are not directly affected. These trends come mostly from intuition, but they can be seen in later results.

We will need equations for the vertex locations and the constraints, but first we should try to qualitatively understand how each change of the geometry will affect the inlet performance. The middle columns of Table 1 show how the two types of constraint are affected by changes in the three inputs and the two main flight condition variables. The last two columns show how two performance metrics are affected.

Table 1. Qualitative trends caused by various inputs.

\begin{tabular}{cccccc}
\hline \hline Variable & $\chi_{1}$ & $\chi_{2}, \ldots \chi_{m}$ & $\chi_{m+1}, \ldots, \chi_{N}$ & $A_{1}$ & $p_{0, N+1} / p_{0,1}$ \\
\hline$u_{1} \uparrow$ & $\downarrow$ & $\downarrow$ & $\downarrow$ & $\uparrow$ & - \\
$u_{2} \downarrow$ & $\uparrow$ & $\downarrow$ & $\uparrow$ & $\uparrow$ & - \\
$u_{3} \uparrow$ & $\downarrow$ & $\downarrow$ & $\uparrow \downarrow$ & $\uparrow$ & $\downarrow$ \\
$M \uparrow$ & $\downarrow$ & $\downarrow$ & $\uparrow$ & $\uparrow$ & $\downarrow$ \\
$\alpha \uparrow$ & $\uparrow \downarrow$ & $\uparrow$ & $\downarrow$ & $\uparrow$ & $\downarrow$ \\
\hline \hline
\end{tabular}

Table 1 shows some useful information about the effectiveness of the three inputs by simply comparing trends among the various parameters. It shows that increasing the Mach number and increasing the angle of attack have opposite effects on $\chi_{1}, \ldots, \chi_{m}$ and $\chi_{m+1}, \ldots, \chi_{N}$. As an increase in $u_{1}$ causes both $\chi_{1}$ and $\chi_{N}$ to decrease, it cannot be a particularly useful parameter to counteract changes in $M$ or $\alpha$. The other two inputs do not have this problem, and they have substantially more potential as a result. Of the remaining two inputs, Table 1 does not give a decisive answer on which is better. The cowl rotation, $u_{3}$, has a more direct effect on the pressure recovery factor; in fact, any nonzero value of $u_{3}$ should decrease the pressure recovery factor. The other caveat here is that the cowl rotation is only useful when $n=2$.

The vertices of the vehicle body are not affected by the variable geometry, so the formula for the first $N$ vertices do not include $u_{1}, u_{2}$, or $u_{3}$. The next vertex, which is the leading edge of the cowl, is affected by all three. The last 


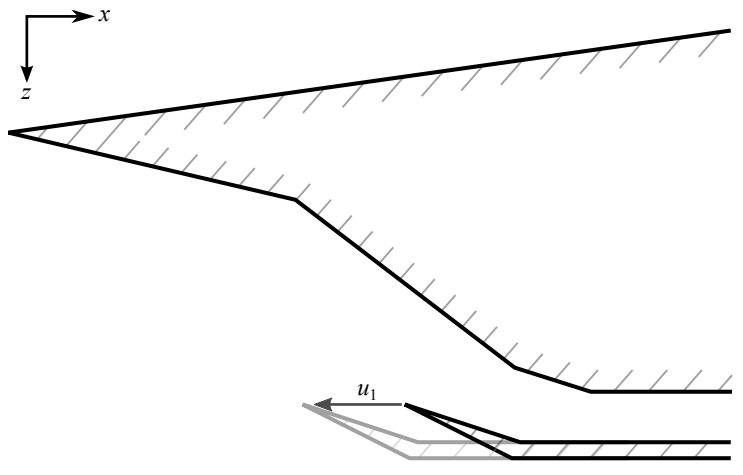

a) Forward deflection, $u_{1}$.

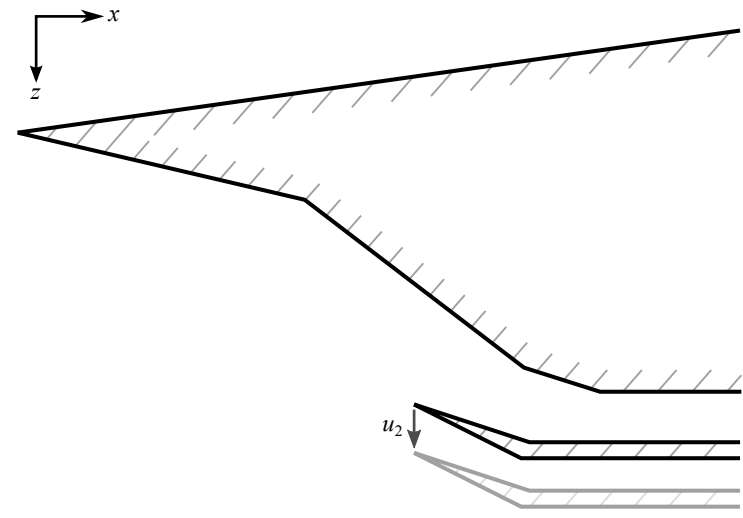

b) Downward deflection, $u_{2}$.

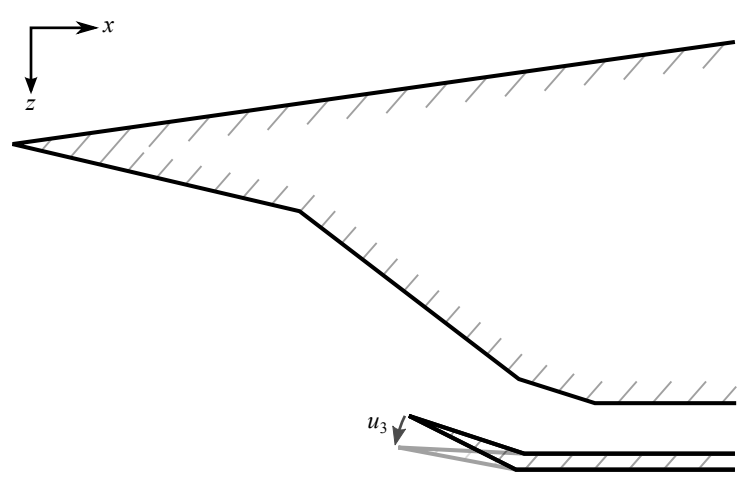

c) Downward rotation, $u_{3}$.

Figure 3. The three degrees of freedom for variable geometry.

$n-1$ vertices are affected by $u_{1}$ and $u_{2}$, although only it can be written in such a way that the inputs do not appear in the equations.

$$
\begin{array}{rlr}
x_{1} & =0 & \\
y_{1} & =0 & \\
x_{i} & =x_{i-1}+\lambda_{i} \cos \bar{\theta}_{i} & \\
z_{i} & =z_{i-1}+\lambda_{i} \sin \bar{\theta}_{i} & i=2, \ldots \\
x_{i} & =1-u_{1}+\lambda_{N}\left(\cos \bar{\theta}_{m+2}-\cos \theta_{m+2}\right) & \\
z_{i}=H+u_{2}+\lambda_{N}\left(\sin \bar{\theta}_{m+2}-\sin \theta_{m+2}\right) & i=N \\
x_{i}=x_{i-1}+\lambda_{i-2} \cos \theta_{i-n} & \\
z_{i}=z_{i-1}+\lambda_{i-2} \sin \theta_{i-n} & i=N
\end{array}
$$

Here $\bar{\theta}_{k}$ is the undeflected flowpath angle, while $\theta_{k}$ accounts for $u_{3}$. The formula is

$$
\theta_{k}=\left\{\begin{array}{cc}
\bar{\theta}_{k} & k \neq m+2 \\
\bar{\theta}_{k}-u_{3} & k=m+2
\end{array}\right.
$$

The lengths used for constraints are

$$
\begin{array}{ll}
\chi_{k}=x_{N+1}-x_{k}-\left(z_{N+1}-z_{k}\right) \cot \sigma_{k} & k=1, \ldots, m \\
\chi_{k}=\frac{z_{k+n}-z_{k}-\left(x_{k+n}-x_{k}\right) \tan \sigma_{k}}{\sin \theta_{k+1}-\tan \sigma_{k} \cos \theta_{k+1}} & k=m+1, \ldots, N
\end{array}
$$


The constraints themselves are

$$
\begin{aligned}
\chi_{m} & \geq 0 \\
\chi_{k} & \geq \chi_{k+1} \\
\chi_{k} & \geq 0 \\
\chi_{k} & \leq \lambda_{k}
\end{aligned}
$$

$$
\begin{aligned}
k & =1, \ldots, m-1 \\
k & =m+1, \ldots, N \\
k & =m+1, \ldots, N-1
\end{aligned}
$$

\section{Design Process}

We now have $2 N+n-2$ degrees of freedom and $N+n-1$ constraints. If we treat all of the constraints as equality constraints, this leaves us with $N-1$ degrees of freedom. The simplest approach is to use the first $N-1$ deflection angles, $\delta, \ldots, \delta_{N-1}$. The last shock angle is constrained because the flow must be parallel to the $x$-axis downstream of the last shock, i.e. $\theta_{N+1}=0$.

We expect that most or all of the inequality constraints in Eqs. (21-24) will be active for an optimal inlet for various reasons. If $\chi_{m}>0$, then we can move the location of the cowl leading edge forward, which will make the inlet capture more air without affecting the compression ratio. We can also see that we would like to keep $\chi_{m+1}$ close to zero because that means less wave interaction, and reducing $\lambda_{m+1}$ will make the inlet smaller without changing the capture area.

These three comments also demonstrate that it is difficult to formalize the inlet design as an optimization problem. This is essentially a result of having three different objective functions: the capture area, the pressure recovery factor, and height of the inlet. The initial design process described here seeks to find an inlet that meets all of the constraints and treats maximization of the pressure recovery factor as the primary objective. Specifically, the deflection angles, $\delta_{1}, \ldots, \delta_{N-1}$ are selected to optimize the pressure recovery factor for one operating condition using a simplified model. The other decision variables, $\lambda_{1}, \ldots, \lambda_{N+n-2}$, are selected so that all constraints are met. In addition, the design takes advantage of any opportunity to increase the capture area as long as it does not decrease the pressure recovery factor.

\section{A. Flight Envelope}

From the introduction to this section, we discussed the advantages of having $\chi_{m+1}=0$ and $\chi_{m+1}=\lambda_{m+1}$. However, this does not mean that using $\lambda_{m+1}=0$ is a good choice. Instead, we require that constraints (23) and (24) are satisfied for a given range of Mach numbers and angles of attack.

Let $\mathcal{T}$ be the set of all pairs of Mach number and angle of attack, $(M, \alpha)$, for which we want the constraints to apply. Now $\chi_{m+1}$ will take a range of values, and we use

$$
\lambda_{k}=\max _{(M, \alpha) \in \mathcal{T}} \chi_{k}(M, \alpha) \quad k=m+1, \ldots, N
$$

to determine the lengths of the internal ramps. To solve this problem in general requires an optimization to find the maximum value of $\chi_{k}$, but often $\mathcal{T}$ is only a curve, where $\alpha$ is given as a function of the Mach number. Because an air-breathing hypersonic vehicle is likely to fly at a constant or nearly constant dynamic pressure [9, 12], the altitude is determined for a given Mach number. Once the altitude and Mach number are fixed, there is a certain angle of attack that will trim the entire vehicle [14]. Of course, we cannot know the trimmed flight conditions before we have designed the vehicle.

As a compromise of performance, robustness, and simplicity, the examples in this paper treat the flight envelope as a rectangle in $(M, \alpha)$-space. We denote the minimum angle of attack as $\alpha^{-}$and the maximum angle of attack as $\alpha^{+}$. Similarly, $M^{-}$and $M^{+}$are the minimum and maximum Mach numbers, respectively. Figure 4 shows a sketch of this type of flight envelope, and Table 2 explains the five labeled flight conditions and identifies which points have the minimum and maximum values for each wave angle.

\section{B. Selection of Shock Angles}

The first task of the design process is to select the deflection angles for external and internal shocks. There are many metrics by which to evaluate inlet performance, but the most important task of the inlet is to provide adequate pressure to the isolator or combustor. Thus the inlet must satisfy an additional constraint, which is that the static pressure at the inlet exit, $p_{N+1}$, must be greater than or equal to a certain value. If two inlets have the same exit pressure, then we would expect the one that has less stagnation pressure loss will generate less drag because it has a higher exit flow 
Table 2. Values for important qualities at five critical flight conditions. A "_" symbol denotes a value that is between the extremes.

\begin{tabular}{cccccc}
\hline \hline Condition & $M$ & $\alpha$ & $\sigma_{1}$ & $\sigma_{2}, \ldots, \sigma_{m}$ & $\sigma_{m+1}, \ldots, \sigma_{N}$ \\
\hline $\mathrm{A}$ & $M^{-}$ & $\alpha^{+}$ & - & $\max$ & $\min$ \\
$\mathrm{B}$ & $M^{+}$ & $\alpha^{+}$ & - & - & - \\
$\mathrm{C}$ & $M^{+}$ & $\alpha^{-}$ & - & $\min$ & $\max$ \\
$\mathrm{D}$ & $M^{-}$ & $\alpha^{-}$ & - & - & - \\
$\mathrm{E}$ & $M^{+}$ & - & $\min$ & - & - \\
\hline \hline
\end{tabular}

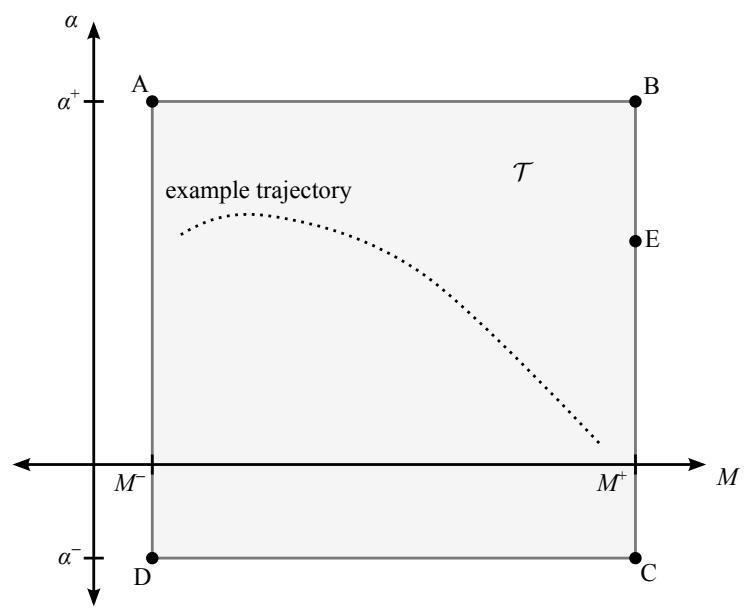

Figure 4. Flight envelope used for inlet design. The envelope $\mathcal{T}$ is the gray region, and the dotted line shows an example trajectory.

velocity. In reality, multidisciplinary optimization of an entire scramjet vehicle would not necessarily select an inlet with minimum drag, but we use pressure recovery factor as the objective function for this inlet design.

With the objective function of maximum pressure recovery factor selected, we must still choose a way to evaluate the pressure recovery factor for the entire envelope $\mathcal{T}$. One example of such a method to evaluate the pressure recovery, which is a scalar, for a range of conditions is to use the average value for all flight conditions in $T$. However, using that evaluation of the pressure recovery as the objective function could result in a design that has excellent performance at some conditions and poor performance at others. Instead, we use the minimum value of the pressure recovery factor as our objective function. In other words, the goal is to design the inlet that has the highest lower bound on the pressure recovery factor. Table 1 tells us that the flight condition with the lowest pressure recovery factor is condition B, which has the maximum Mach number and maximum angle of attack.

In addition, we would like to consider the pressure constraint only at a single point. Although the compression ratio, $\varepsilon=p_{N+1} / p_{1}$, increases with higher Mach numbers, these vehicles tend to fly on trajectories with approximately constant dynamic pressure, wich causes the freestream pressure to decrease as the Mach number increases. Usually, this decrease is faster than the increase in $\varepsilon$, so we use

$$
\frac{p_{N+1}^{\mathrm{C}}}{p_{1}^{\mathrm{C}}} \geq \varepsilon
$$

as the compression constraint. This ensures that the static pressure at the inlet exit will not be below $\varepsilon p_{1}\left(M^{+}\right)$for any condition in $\mathcal{T}$.

Smart [12] showed that the pressure recovery factor is optimized when the external shocks have very nearly equal normal Mach numbers. The same could be said for the internal shocks. To simplify the presentation and avoid reproducing work on optimization for such inlets, we have assumed that the two sets of normal Mach numbers are exactly equal. Conveniently, the compression ratio across a single oblique shocks can be written directly in terms of only the normal Mach number, so we write the compression ratio across each shock as

$$
\varepsilon_{k}=\frac{p_{k+1}}{p_{k}}=\frac{2 \gamma}{\gamma+1} M_{k}^{2} \sin ^{2} \beta_{k}-\frac{\gamma-1}{\gamma+1}
$$


Then the pressure constraint is

$$
\varepsilon=\prod_{k=1}^{N} \varepsilon_{k}^{\mathrm{C}}
$$

The result that we are using equal normal Mach numbers means that

$$
\varepsilon_{1}^{\mathrm{B}}=\ldots=\varepsilon_{m}^{\mathrm{B}} \quad \varepsilon_{m+1}^{\mathrm{B}}=\ldots=\varepsilon_{N}^{\mathrm{B}}
$$

and these are the two independent variables used in the design. Once values for $\varepsilon_{1}^{\mathrm{B}}$ and $\varepsilon_{N}^{\mathrm{B}}$ have been chosen, all of the deflection angles, $\delta_{1}, \ldots, \delta_{N}$ can be found. These can be used to evaluate the exit flowpath angle, $\theta_{N+1}$, and the compression constraint at condition $\mathrm{C}$. If both of these values are correct, then $\varepsilon_{1}^{\mathrm{B}}$ and $\varepsilon_{N}^{\mathrm{B}}$ are correct, which means $\delta_{1}, \ldots, \delta_{N}$ are also correct.

\section{Exterior Inlet Geometry}

The next task is to determine the height of the cowl leading edge, $H$, and the lengths of the exterior panels. These values must be chosen so that constraints (21) and (22) are satisfied, i.e. $\chi_{1} \geq \ldots \geq \chi_{m} \geq 0$, for all conditions in $\mathcal{T}$. Each of these constraint values decreases with increasing Mach number, so we can use $M^{+}$for the design. However, $\chi_{1}$ can either increase or decrease with increasing angle of attack, depending on $M_{1}$ and $\delta_{1}$, so we need to find the angle of attack that leads to the smallest value of $\sigma_{1}$. The condition corresponding to this value is condition $\mathrm{E}$ in Table 2 and Fig. 4.

The angle of the first wave is simply

$$
\sigma_{1}=\beta_{1}-\alpha
$$

so minimizing $\sigma_{1}$ is equivalent to finding the point where $\partial \beta_{1} / \partial \alpha=1$. This has a solution when

$$
\sin ^{2} \beta_{1}^{\mathrm{E}}=\frac{1}{2+\frac{\gamma-1}{2}\left(M^{+}\right)^{2}}
$$

This value is used to find the actual angle of attack using

$$
\tan \left(\alpha^{\mathrm{E}}+\theta_{2}\right)=\cot \beta_{1}^{\mathrm{E}} \frac{\left(M^{+}\right)^{2} \sin ^{2} \beta_{1}^{\mathrm{E}}-1}{\frac{\gamma+1}{2}\left(M^{+}\right)^{2}-\left(M^{+}\right)^{2} \sin ^{2} \beta_{1}^{\mathrm{E}}+1}
$$

If this value of $\alpha^{\mathrm{E}}$ is outside the range of angles of attack, $\left[\alpha^{-}, \alpha^{+}\right]$, use whichever value is closer.

With the value of $\sigma_{1}^{\mathrm{E}}=\beta_{1}^{\mathrm{E}}-\alpha^{\mathrm{E}}$ determined, the next step is to calculate the vertical position of the cowl leading edge, $H$. If there is only a single external shock, i.e. $m=1$, then

$$
H=\tan \sigma_{1}^{\mathrm{E}}
$$

If $m>1$, then

$$
\begin{aligned}
& \lambda_{1}=\left(1+\frac{\tan \sigma_{1}^{\mathrm{E}}-\tan \theta_{2}}{\tan \sigma_{2}^{\mathrm{E}}-\tan \sigma_{1}^{\mathrm{E}}} \frac{\tan \sigma_{2}^{\mathrm{E}}}{\tan \sigma_{2}^{\mathrm{C}}}\right)^{-1} \sec \theta_{2} \\
& H=\frac{\tan \sigma_{2}^{\mathrm{E}}-\tan \theta_{2}}{\tan \sigma_{2}^{\mathrm{E}}-\tan \sigma_{1}^{\mathrm{E}} \lambda_{1} \cos \theta_{2} \tan \sigma_{1}^{\mathrm{E}}}
\end{aligned}
$$

The reason for the differing approaches depending on the value of $m$ is that the condition $\chi_{1} \geq \chi_{2}$ does not exist when $m=1$. The subsequent panel lengths are calculated using

$$
\lambda_{k}=\frac{\left(1-x_{k-1}\right) \tan \sigma_{k}^{\mathrm{C}}-H+z_{k-1}}{\tan \sigma_{k}^{\mathrm{C}}-\sin \theta_{k}} \quad k=2, \ldots, m-1
$$

in combination with equation (12) and (13).

According to the simplified analysis of the inlet as simply a sequence of shocks, none of the values of $H$ or $\lambda_{1}, \ldots, \lambda_{m-1}$ has a direct effect on the pressure recovery factor. Rather, the formulas in this subsection provide the values that maximizes capture are while ensuring that the simplified analysis is approximately correct. 


\section{Interior Inlet Geometry}

When considering of the internal portion of the inlet, i.e. shocks $m+1, \ldots, N$, the design should also take full advantage of the variable geometry inputs, $u_{1}$ and $u_{2}$. The third input, $u_{3}$, is ignored because the simplified analysis predicts that any nonzero value of $u_{3}$ will lower the pressure recovery factor.

The first task is to locate the inlet shoulder, that is, point $m+1$, which is equivalent to finding the value of $\lambda_{m}$. In order to ensure that $\chi_{m+1} \geq 0$ for all conditions, the value of $\lambda_{m}$ must be sufficiently small. To minimize the amount of interaction among the shocks, we want to use the largest value of $\lambda_{m}$ that satisfies all of the constraints. For each condition in $\mathcal{T}$, let

$$
\lambda_{m}^{*}=\frac{H+U_{2}^{*}-z_{m}-\left(1-x_{m}\right) \tan \sigma_{m+1}^{*}}{\sin \theta_{m+1}-\cos \theta_{m+1} \tan \sigma_{m+1}^{*}}
$$

The value of $U_{2}^{*}$ is chosen to be the largest allowable value. The formula is

$$
U_{2}^{*}=\min \left\{U_{2}, z_{m}+\left(1-x_{m}\right) \tan \sigma_{m}^{*}-H, \frac{x_{2} \tan \sigma_{2}^{*}-z_{2}}{\tan \sigma_{2}^{*}-\tan \sigma_{1}^{*}} \tan \sigma_{1}^{*}-H\right\}
$$

where $U_{2}$ is a physical upper limit for how far the cowl can be moved downward. The other two limits come from the constraint $\chi_{m} \geq 0$ and $\chi_{1} \geq \chi_{2}$, respectively. Figure 5 gives a graphical explanation of how $U_{2}^{*}$ is determined. The final value for $\lambda_{m}$ is then

$$
\lambda_{m}=\min \left\{\lambda_{m}^{\mathrm{A}}, \lambda_{m}^{\mathrm{B}}, \lambda_{m}^{\mathrm{C}}, \lambda_{m}^{\mathrm{D}}, \lambda_{m}^{\mathrm{E}}\right\}
$$

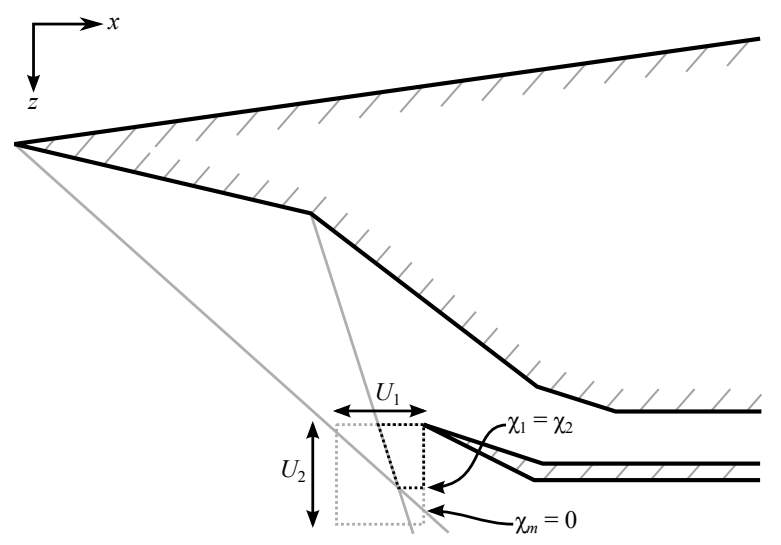

Figure 5. Sketch of the limits for the inputs $u_{1}$ and $u_{2}$. The light dotted box shows the largest inputs that are allowed by the actuators, and the darker dotted shape, whose lower right corner corresponds to $U_{2}^{*}$, is the region of inputs that satisfy all constraints.

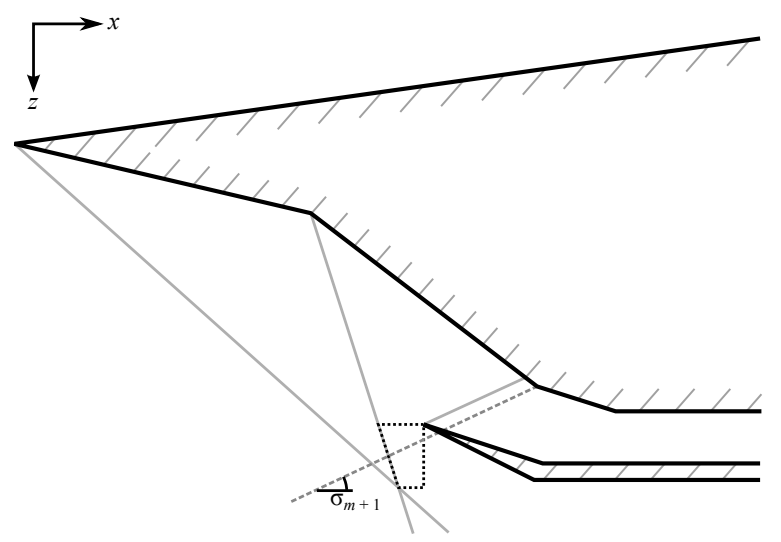

Figure 6. Visual description of the process to choose $u_{1}$ and $u_{2}$ for a given flight condition. If the leading edge is along the dashed line, then $\chi_{m+1}=0$. The dotted trapezoid shows the allowable values for $u_{1}$ and $u_{2}$. 
The next task is to determine the best choice of $u_{1}$ and $u_{2}$ for each operating condition. When we were determining the value of $\lambda_{m}$ in the previous paragraphs, we simply assumed $u_{1}=0$ and $u_{2}=U_{2}^{*}$. This ensured that the first internal shock did not go upstream of point $m+1$, i.e. $\chi_{m+1} \geq 0$. However, the final values of $u_{1}$ and $u_{2}$ for each condition should be chosen to keep $\chi_{m+1}$ as small as possible, which minimizes wave interactions, and to capture as much air as possible. In keeping with our treatment of the pressure recovery factor as the first objective, $u_{1}$ and $u_{2}$ are chosen so that $\chi_{m+1}$ is minimized if it is not possible to satisfy $\chi_{m+1}=0$, and capture area is only used to decide among multiple configurations where $\chi_{m+1}=0$.

Figure 6 shows an example of how this process works. For a given condition $*$, all the wave angles are known. Although shock $m+1$ originates from the cowl leading edge, we draw this shock backwards from the shoulder, i.e. point $m+1$. In Fig. 6, this hypothesized shock is the dashed gray line. If $u_{1}$ and $u_{2}$ are chosen so that the leading edge of the cowl lies somewhere along this line, then it will be an actual shock, and $\chi_{m+1}=0$. Thus, selecting $u_{1}$ and $u_{2}$ comes down to finding intersections between this hypothesized shock and the dotted box that gives the limits of the allowable values for $u_{1}$ and $u_{2}$. Depending on the exact configuration, there may not be any such intersections, but in this case, the process used to select $\lambda_{m}$ ensures that the value of $\chi_{m+1}$ will be positive.

For a given condition $*$, the value of $u_{2}$ is

$$
\begin{aligned}
u_{2}^{*}=\max \left\{0, \min \left\{U_{2}^{*}, z_{m+1}+\left(1-U_{1}-x_{m+1}\right) \tan \sigma_{m+1}-H,\right.\right. & \\
& \left.\left.\frac{z_{m+1} \tan \sigma_{m}^{*}-z_{m} \tan \sigma_{m+1}^{*}-\left(x_{m+1}-x_{m}\right) \tan \sigma_{m}^{*} \tan \sigma_{m+1}^{*}}{\tan \sigma_{m}^{*}-\tan \sigma_{m+1}^{*}}-H\right\}\right\}
\end{aligned}
$$

which is the result of finding the intersection of shock $m$ and the hypothetical dashed line from Fig. 6 . Then the other input can be found using

$$
u_{1}^{*}=1-\max \left\{x_{m+1}+\left(H+u_{2}^{*}-z_{m+1}\right) \cot \sigma_{m+1}^{*}, x_{m}+\left(H+u_{2}^{*}-z_{m}\right) \cot \sigma_{m}^{*}\right\}
$$

Once the cowl has been placed, the only remaining task is to decide the lengths of the interior panels, $m+1$ through $m+2 n-2$. If $n=1$, i.e. there is only one internal shock, then there are no internal panels, and the design is completed. Otherwise the following process is used for $k=m+1, \ldots, N-1$.

$$
\begin{aligned}
\lambda_{k} & =\max \left\{\chi_{k}^{\mathrm{A}}, \chi_{k}^{\mathrm{B}}, \chi_{k}^{\mathrm{C}}, \chi_{k}^{\mathrm{D}}, \chi_{k}^{\mathrm{E}}\right\} \\
\lambda_{k+n-1}^{*} & =\frac{z_{k+n}^{*}-z_{k+1}+\left(x_{k+n}^{*}-x_{k+1}\right) \tan \sigma_{k+1}^{*}}{\sin \theta_{k+1}-\cos \theta_{k+1} \tan \sigma_{k+1}^{*}} \\
\lambda_{k+n-1} & =\max \left\{\lambda_{k+n-1}^{\mathrm{A}}, \lambda_{k+n-1}^{\mathrm{B}}, \lambda_{k+n-1}^{\mathrm{C}}, \lambda_{k+n-1}^{\mathrm{D}}, \lambda_{k+n-1}^{\mathrm{E}}\right\}
\end{aligned}
$$

\section{Results}

The evaluation of this design method is broken into three main parts. Section IV.A attempts to determine how much benefit a variable-geometry design can provide compared to a fixed-geometry design. Since this discussion does not address how difficult a variable-geometry would be to physically construct and implement, predicting how much the design would benefit from variable geometry is the primary motivation for this paper. The second subsection explores what values of $u_{1}$ and $u_{2}$ are most effective with the goal of showing that Eqs. (40) and (41) give useful results. The last subsection gives some guidance on which inputs are more effective for the case that only one variable-geometry input is allowed.

To accomplish these goals, five separate inlet designs are used. All of them have geometries determined using the methods described in Section III, but using different values for the design parameters. They range from a fixed geometry that is only designed to operate at one condition to a design that operates over a wider range of conditions using all variable-geometry parameters. In each case, there are two external shocks $(m=2)$ and two internal shocks $(n=2)$. Table 2 summarizes the five designs.

The range of conditions used for the design process has a relatively wide range of Mach numbers (2) and a relatively narrow range of angle of attack $\left(1.5^{\circ}\right)$. Although this study did not simulate a complete vehicle and therefore does not consider trim, previous studies [14,15] have shown that the angle of attack changes little as a function of Mach number for some vehicles.

\section{A. Evaluation of Variable Geometry}

Figures 7 and 8 show the performance of three different inlet designs. The first design, labeled "Single-condition" in the plots, is designed using $M^{-}=M^{+}=8$ and $\alpha^{-}=\alpha^{+}=0$. In other words, this design is optimized for 
Table 3. List of parameters used for sample designs.

\begin{tabular}{l|cc|cc|cc}
\hline \hline LABEL & $M^{-}$ & $M^{+}$ & $\alpha^{-}$ & $\alpha^{+}$ & $U_{1}$ & $U_{2}$ \\
\hline Single-condition & 8 & 8 & $0^{\circ}$ & $0^{\circ}$ & 0 & 0 \\
Fixed geometry & 7 & 9 & $-0.5^{\circ}$ & $1^{\circ}$ & 0 & 0 \\
Variable geometry & 7 & 9 & $-0.5^{\circ}$ & $1^{\circ}$ & 0.1 & 0.015 \\
Horizontal only & 7 & 9 & $-0.5^{\circ}$ & $1^{\circ}$ & 0.1 & 0 \\
Vertical only & 7 & 9 & $-0.5^{\circ}$ & $1^{\circ}$ & 0 & 0.015 \\
\hline \hline
\end{tabular}

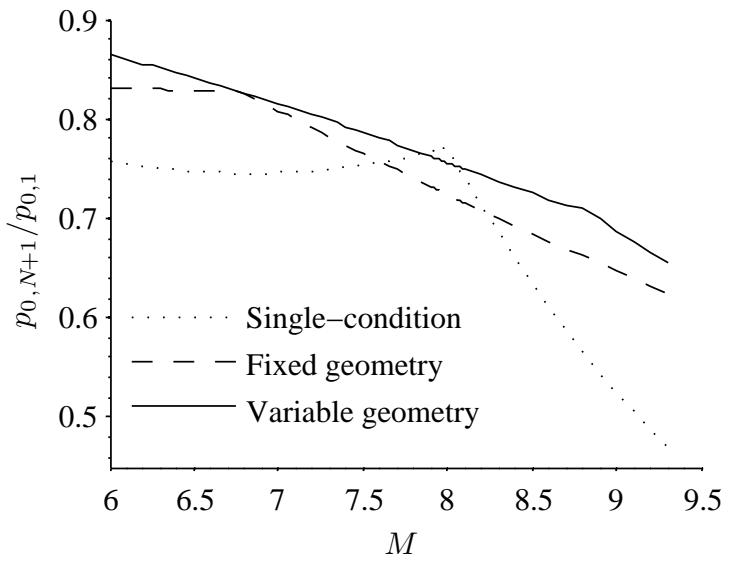

a) Pressure recovery factor.

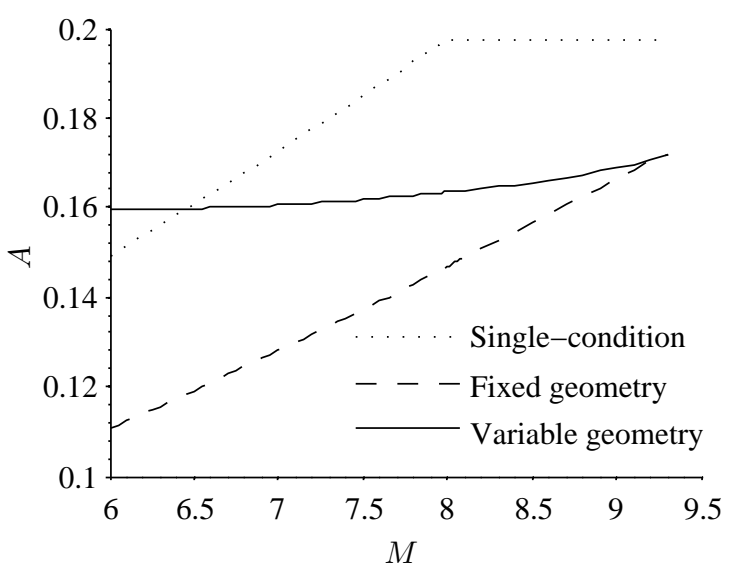

c) Capture area.

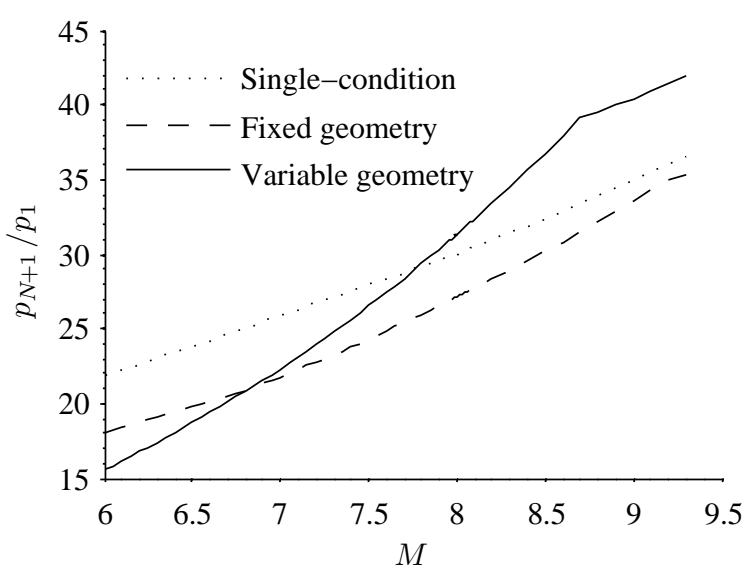

b) Compression ratio.

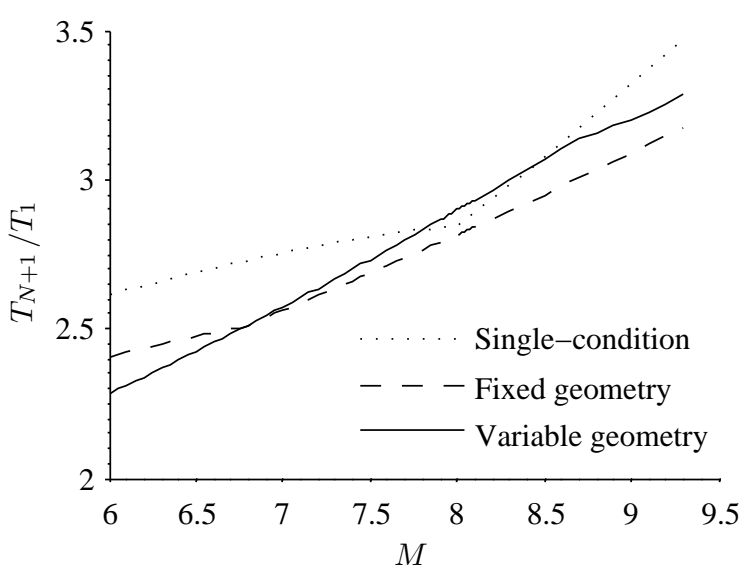

d) Temperature ratio.

Figure 7. Performance of three inlet designs operating at $\alpha=0$ and a range of Mach numbers.

a single flight condition, and no consideration is taken for the robustness of this design. The second design, labeled "Fixed geometry", is designed for a Mach number ranging from 7 to 9 and angles of attack between $-0.5^{\circ}$ and $1^{\circ}$. However, the inputs are all restricted to 0 , so this is essentially the same design as the one used in [4]. The third design, labeled "Variable geometry", uses the same conditions but the cowl is allowed to move. Table 3 gives a summary of the parameters used for each of the three designs and two more designs used in a later figure.

As expected, the single-condition design is the best-performing inlet at its design condition of $M=8$ and $\alpha=0$. However, the performance of the single-condition inlet falls off sharply for other Mach numbers. In addition, there is a sharp corner in most of the performance metrics at $M=8$ and $\alpha=0$, so the inlet would be very difficult to control around the design condition. Both of the other designs avoid these problems and have a smooth, monotonic performance throughout the design region.

What is more relevant to this paper is the difference in absolute performance between the "Fixed geometry" and 


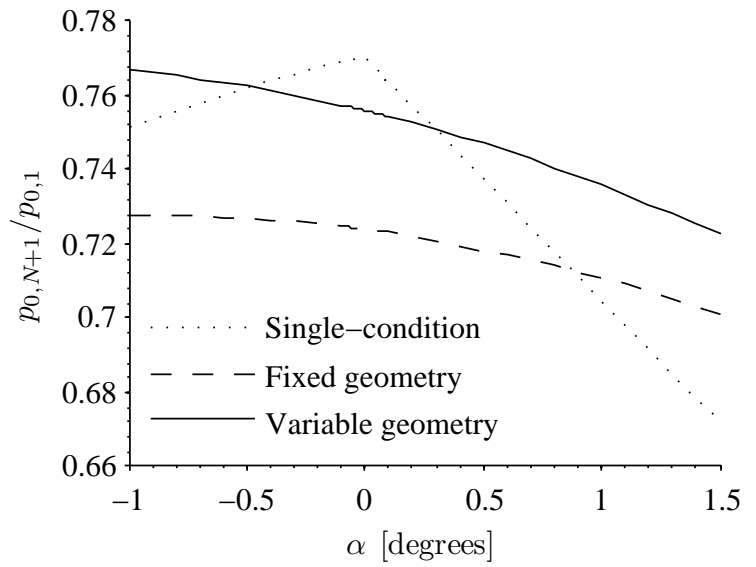

a) Pressure recovery factor.

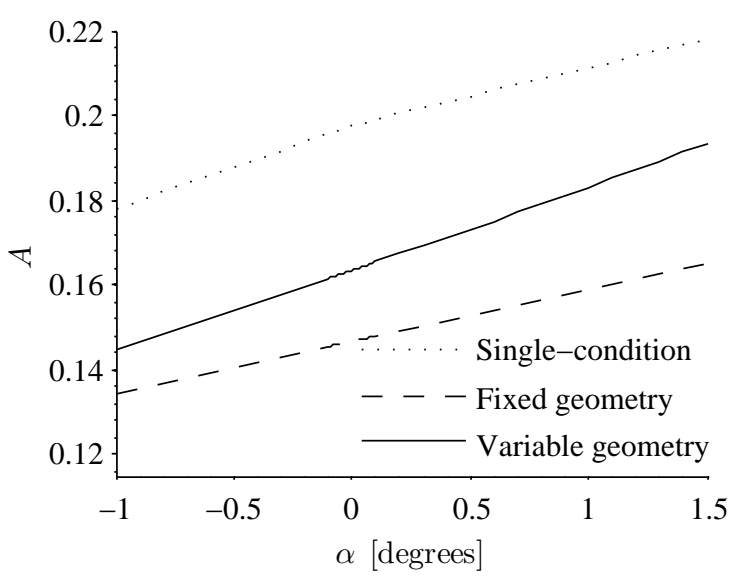

c) Capture area.

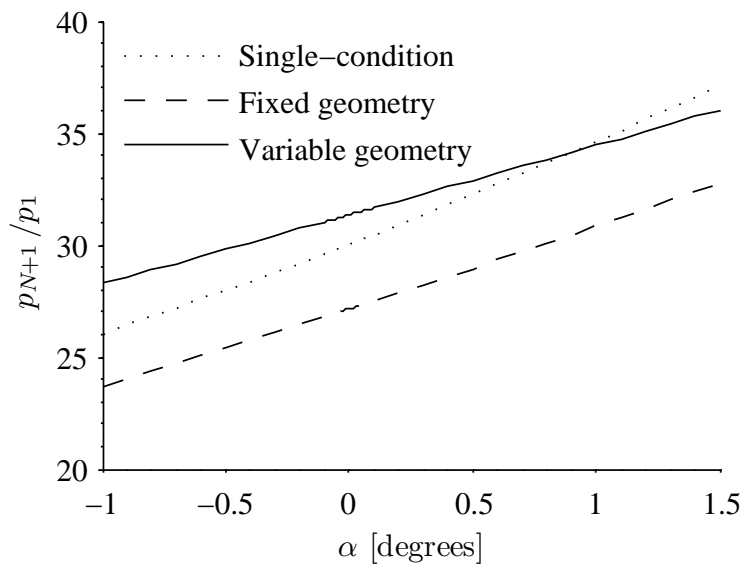

b) Compression ratio.

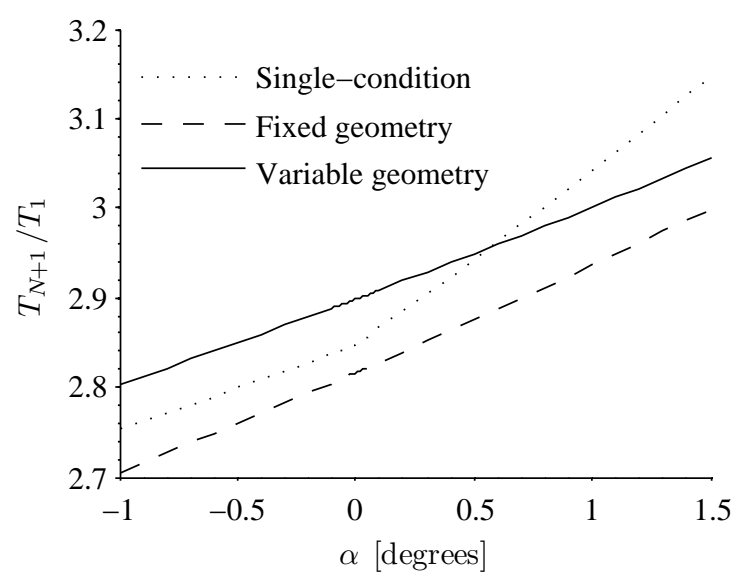

d) Temperature ratio.

Figure 8. Performance of three inlet designs operating at $M_{1}=8$ and a range of angles of attack.

"Variable geometry" designs. Throughout the design range, the variable-geometry design performs as well as or better than the fixed-geometry design in every performance metric at every flight condition. The primary reason for this improved performance is that a movable cowl alleviates some of the conflicting requirements of operating over a range of conditions. For example, Fig. 7a shows that both designs have the similar pressure recovery factors at $M=7$, but when $M=9$, the fixed-geometry inlet cannot do anything to prevent the increased shock interactions. Similarly, Fig. 7c shows that both designs have similar area capture at $M=9$, but the fixed-geometry inlet is powerless to capture more area for lower Mach numbers. For a fixed geometry, the cowl position is constrained by the maximum Mach number condition, and as a result the cowl will be far away from its constraints at the minimum Mach number condition. If the cowl is simply allowed to move, it can be much closer to its ideal position for all flight conditions.

The comparison between these three designs shows that there is a large potential benefit if a variable-geometry inlet can be constructed.

\section{B. Selection of Inputs}

The design algorithms in Section III include suggested values for the control variables $u_{1}$ and $u_{2}$. The equations, (40) and (41), give a value for $u_{1}$ and $u_{2}$ that is based on a simplified model. They are intended to maximize the pressure recovery factor while taking into account the area capture if two or more values would give the same pressure recovery factor. In this section, we use the more sophisticated method (SAMURI) to see if the results differ from the simplified model.

To address the claim that Eqs. (40) and (41) accomplish this goal, Fig. 9 plots the pressure recovery factor and area capture as a function of the control inputs. In each plot, two of the input variables are changed while the third is kept 
constant at its value suggested by Eqs. (40) and (41). Although not explicitly in the equations, the default value for $u_{3}$ is always zero. In Fig. 9, the flight condition is kept constant at $M=8$ and $\alpha=0$.

The small " $\times$ " in each plot shows the inputs suggested by the design algorithm in Section III. In all three plots of the pressure recovery factor (Figs. 9a, 9c, and 9e), the symbol is very close to the optimum pressure recovery factor. In Figs. $9 \mathrm{a}$ and $9 \mathrm{~b}$, we can see that slightly decreasing both $u_{1}$ and $u_{2}$ would increase the pressure recovery factor very slightly, but doing so would be at a direct-and much more significant-loss of capture area.

These results indicate that Eqs. (40) and (41) properly identify the correct position for the inlet cowl given the current inlet design. In particular, the plots appear to justify the claim that the cowl rotation, $u_{3}$, should not be used. However, we argued that the values of $u_{1}$ and $u_{2}$ should be incorporated into the design process, and the current inlet design assumes that $u_{1}$ and $u_{2}$ vary according to Eqs. (40) and (41). An optimization that considers $u_{1}, u_{2}, u_{3}$, and the design parameters, $\lambda_{1}, \ldots, \lambda_{N+n-2}, \theta_{1}, \ldots, \theta_{N-1}$, simultaneously may produce a higher pressure recovery factor using nonzero values of $u_{3}$.

Figure 10 shows the values of $u_{1}$ and $u_{2}$ from Eqs. (40) and (41) throughout the design flight envelope. It shows that the trend is not exactly linear, and that the nominal inputs should be calculated using the process described in Section III.

In practical flight, the control system cannot know the exact flight condition, and the control system would not be able to actuate the cowl position infinitely fast. As a result, the inlet must be able to operate over a small range of conditions with a given fixed value of $u_{1}$ and $u_{2}$. The current design does not address this problem, but doing so would only require small changes to Section III. Instead of using constraints such as $\chi_{m} \geq 0$, some nonzero minimum value, call it $\chi^{-}$, should be used. The constraints would become

$$
\begin{aligned}
\chi_{m} & \geq \chi^{-} & & \\
\chi_{k} & \geq \chi_{k+1}+\chi^{-} & k & =1, \ldots, m-1 \\
\chi_{k} & \geq \chi^{-} & k & =m+1, \ldots, N \\
\chi_{k} & \leq \lambda_{k}-\chi^{-} & k & =m+1, \ldots, N-1
\end{aligned}
$$

\section{Comparison of Inputs}

While Section IV.A showed that there is notable potential benefit to a variable-geometry design using $u_{1}$ and $u_{2}$, it would be useful to determine how much utility a single variable-geometry parameter could provide. Figure 11 compares the performance of the bottom three designs from Table 3. One of the designs, labeled "Variable geometry," was present in Figs. 7 and 8. The other two designs allow either only $u_{1}$ or only $u_{2}$ to be nonzero. The "Horizontal only" design has $u_{2}=U_{2}=0$ for all flight conditions, and the "Vertical only" design has $u_{1}=U_{1}=0$.

As predicted using Table 1, allowing the cowl to move forward and backward does not provide much advantage over the fixed-geometry design. On the other hand, allowing the cowl to move vertically gives about half of the advantage using the full "Variable geometry" design.

\section{Conclusions}

A low-order fundamental model was used to evaluate a variable-geometry hypersonic inlet design. The method considers oblique shock waves, expansion fans, shock reflections, and wave interactions. The investigation included over 1500 simulations, each of which took about one second on a $3.3 \mathrm{GHz}$ processor.

We presented an inlet design methodology to effectively utilize variable geometry. Simply adding variable geometry to a previous fixed-geometry design would be much less beneficial. The proposed design algorithm is unified in such a way that fixed-geometry, single-degree-of-freedom, or two-degree-of-freedom designs can be obtained using the same method. Additionally, the design algorithm includes an operating map for the values that the variablegeometry inputs should take as a function of the flight condition. Two metrics were used to determine these inputs, and the suggested values were shown to give good performance in both metrics.

Investigating flight at a range of conditions shows that variable geometry can strongly improve inlet performance. Comparing two inlets designed for the same range of flight conditions, we found that none of the performance metrics was affected negatively at any condition within the design range. One of the three suggested input parameters, namely a rotation of the first panel of the inlet cowl, was found to have no positive effect on performance. Although having the ability to move the cowl both forward and downward was shown to assist inlet performance significantly, consideration was made for the case that only one of the two deflections was available. In this case, the vertical displacement was found to be far more advantageous. 


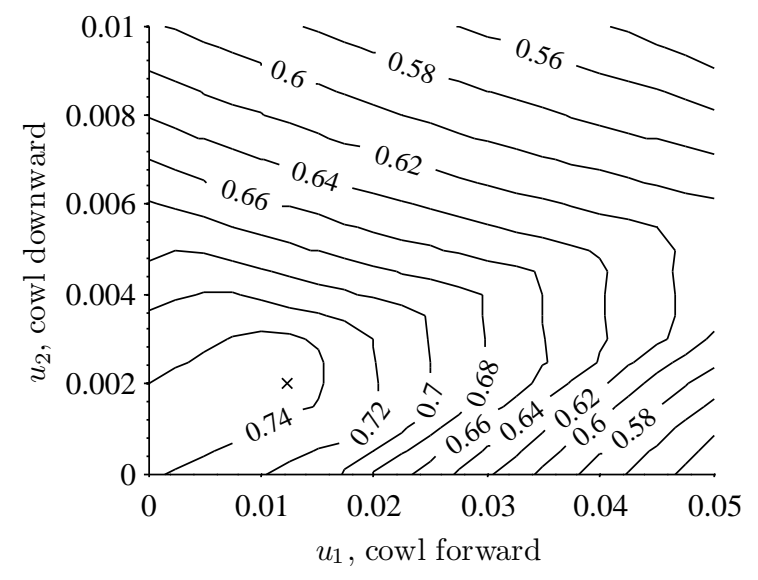

a) Pressure recovery factor in terms of $u_{1}$ and $u_{2}$.

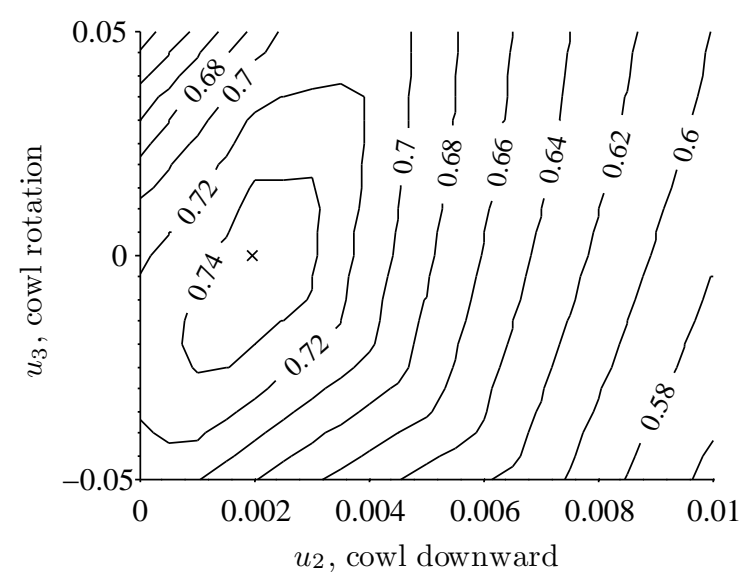

c) Pressure recovery factor in terms of $u_{2}$ and $u_{3}$.

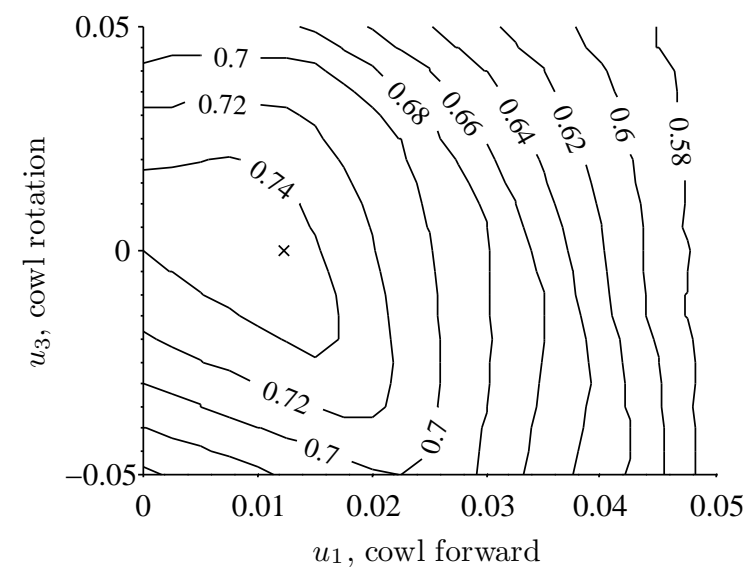

e) Pressure recovery factor in terms of $u_{1}$ and $u_{3}$.

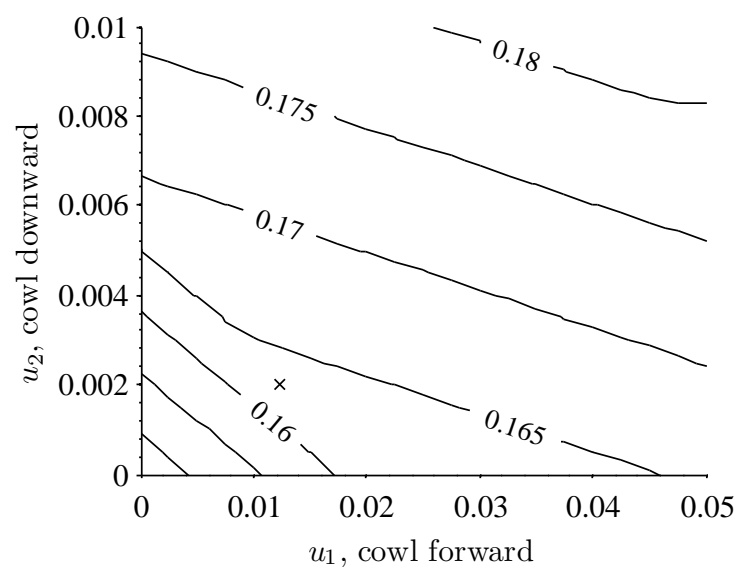

b) Capture area in terms of $u_{1}$ and $u_{2}$.

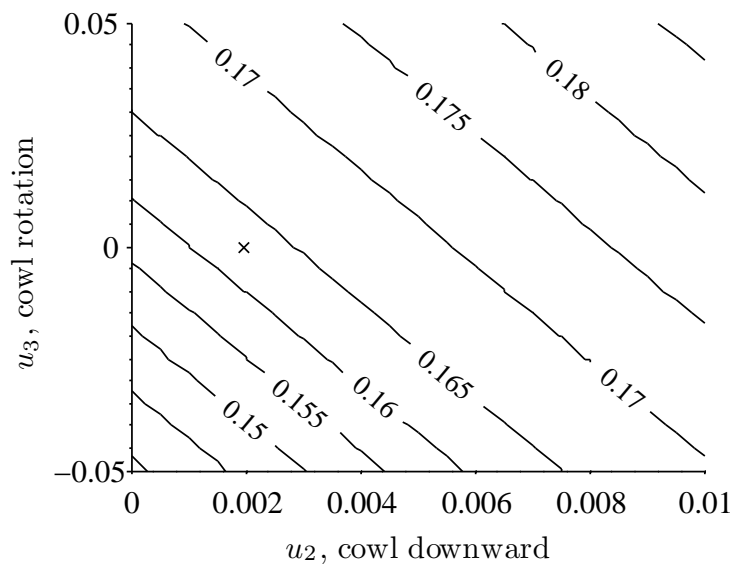

d) Capture area in terms of $u_{2}$ and $u_{3}$.

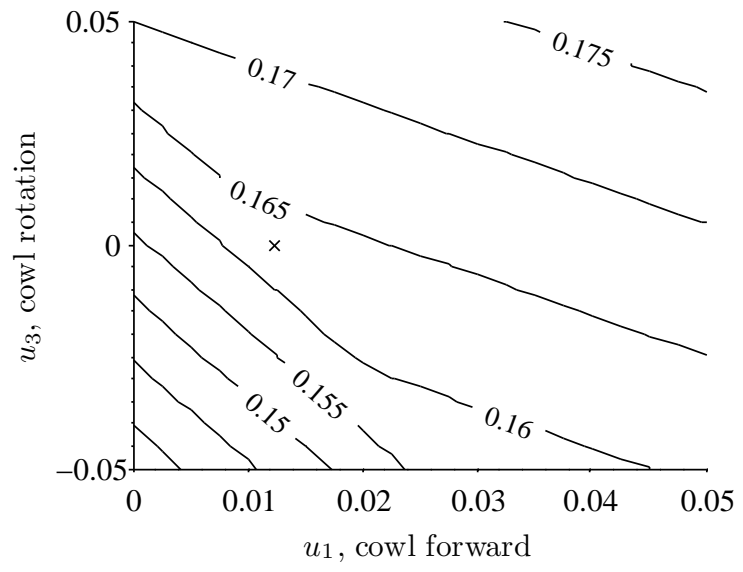

f) Capture area in terms of $u_{1}$ and $u_{3}$,

Figure 9. Performance of variable-geometry inlet design at $M=8$ and $\alpha=0$ for a range of inputs. The $\times$ symbol marks the inputs from Eqs. (40) and (41). 


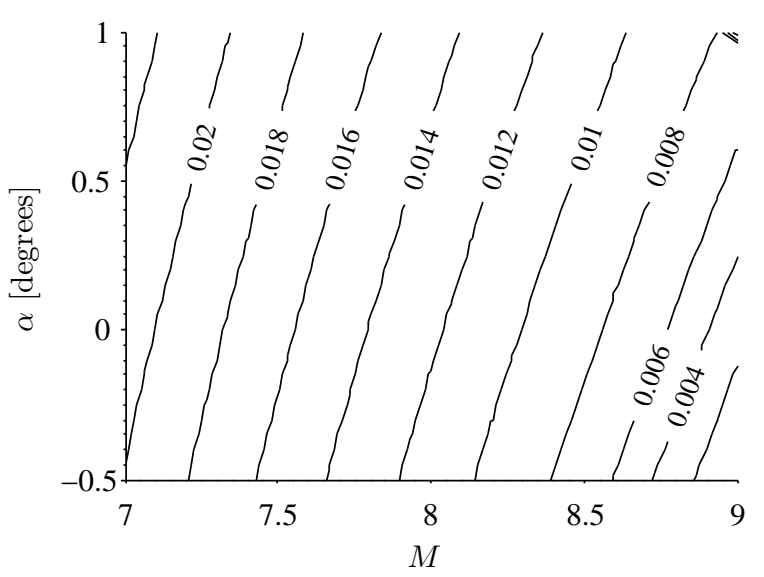

a) Forward cowl deflection, $u_{1}$.

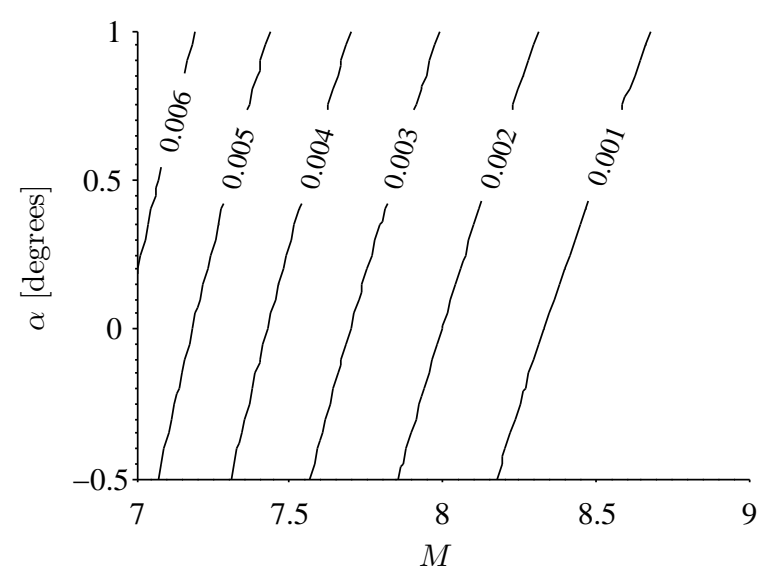

b) Downward cowl deflection, $u_{2}$.

Figure 10. Inputs $u_{1}$ and $u_{2}$ as a function of Mach number and angle of attack using Eqs. (40) and (41).

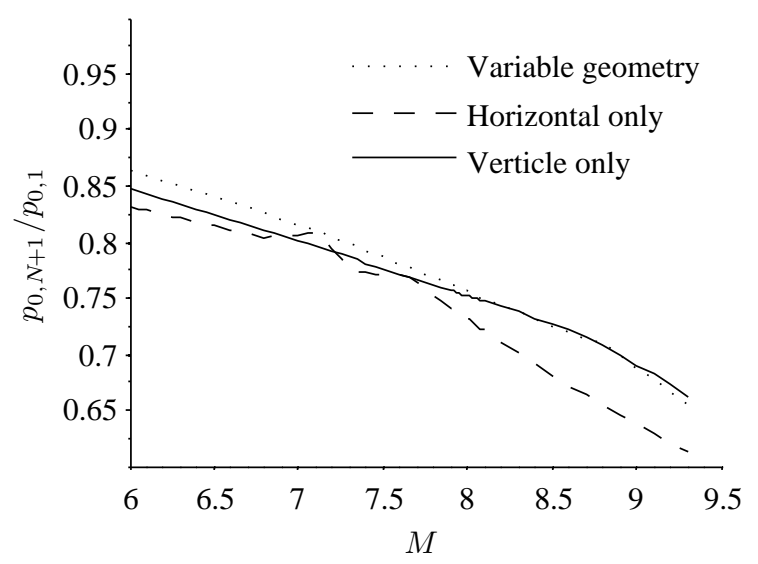

a) Pressure recovery factor.

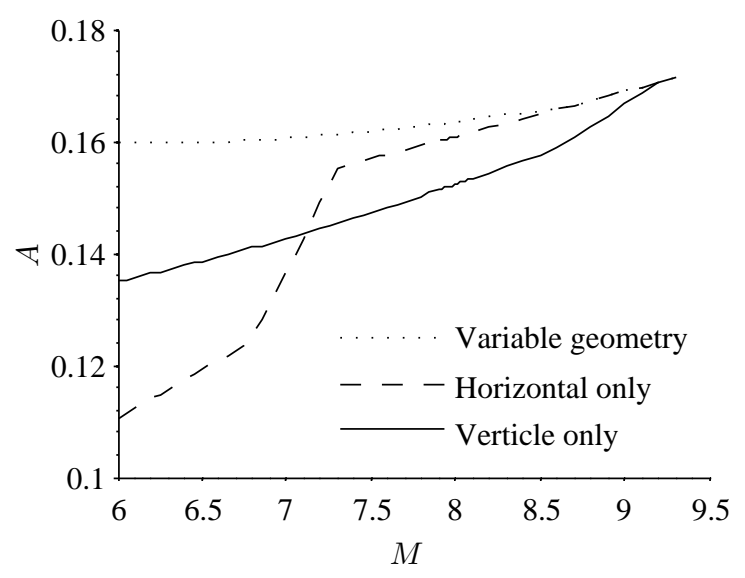

c) Capture area.

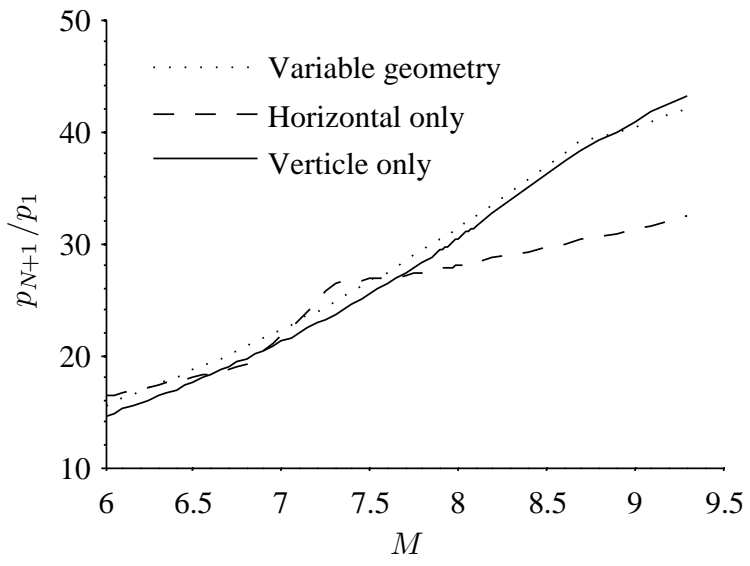

b) Compression ratio.

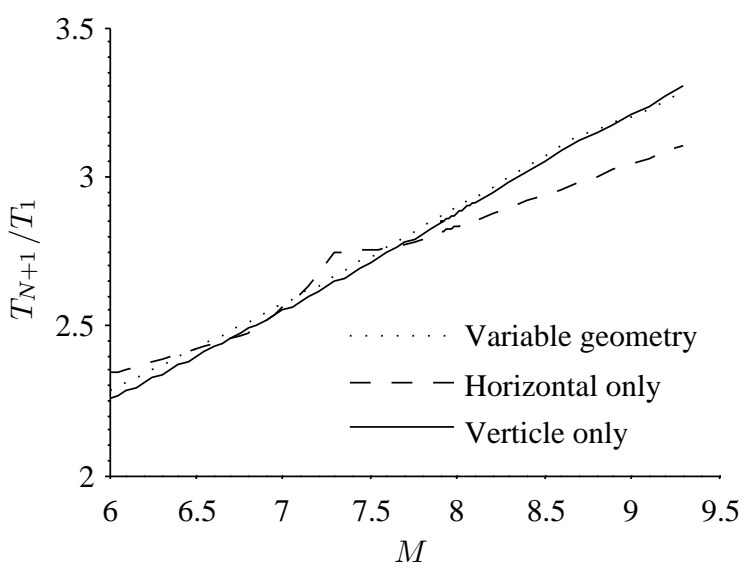

d) Temperature ratio.

Figure 11. Performance of inlet designs using only $u_{1}$, only $u_{2}$, or both $u_{1}$ and $u_{2}$ operating at $\alpha=0$ and a range of Mach numbers. 
The paper only considered the possible benefits of variable geometry and avoided practical considerations such as added weight, difficulty to construct, and increased probability of failure. As a result, the overall benefit of variable geometry has not been evaluated. However, we have shown that a variable-geometry inlet has the potential to provide a marked improvement in the performance of the inlet considered in isolation.

\section{Acknowledgments}

This research is funded by the Air Force Research Laboratory/Air Vehicles Directorate grant FA 8650-07-2-3744 for the Michigan/AFRL Collaborative Center in Control Sciences (Michael Bolender as technical monitor).

\section{References}

[1] Kazmar, R. R., "Airbreathing Hypersonic Propulsion at Pratt \& Whitney - Overview," AIAA/CIRA 13th International Space Planes and Hypersonics Systems and Technologies, 2005, AIAA Paper 2005-3256.

[2] Dalle, D. J., Fotia, M. L., and Driscoll, J. F., "Reduced-Order Modeling of Two-Dimensional Supersonic Flows with Applications to Scramjet Inlets," Journal of Propulsion and Power, Vol. 26, No. 3, 2010, pp. 545-555.

[3] Torrez, S. M., Driscoll, J. F., Dalle, D. J., Bolender, M. A., and Doman, D. B., "Hypersonic Vehicle Thrust Sensitivity to Angle of Attack and Mach Number," AIAA Atmospheric Flight Mechanics Conference, 2009, AIAA Paper 2009-6152.

[4] Torrez, S. M., Driscoll, J. F., Dalle, D. J., and Fotia, M. L., "Preliminary Design Methodology for Hypersonic Engine Flowpaths," 16th AIAA/DLR/DGLR International Space Planes and Hypersonic Systems and Technologies Conference, 2009, AIAA Paper 2009-7289.

[5] Hank, J. M., Murphy, J. S., and Mutzman, R. C., "The X-51A Scramjet Engine Flight Demonstration Program,” 15th AIAA International Space Planes and Hypersonic Systems and Technologies Conference, 2008, AIAA Paper 2008-2540.

[6] Moses, P. L., "X-43C Plans and Status," 12th AIAA International Space Planes and Hypersonic Systems and Technologies, 2003, AIAA Paper 2003-7084.

[7] Emami, S., Trexler, C. A., Auslender, A. H., and Weidner, J. P., "Experimental Investigation of Inlet-Combustor Isolators for a Dual-Mode Scramjet at a Mach Number of 4,” NASA Technical Paper 3502, May 1995.

[8] Starkey, R. P., “Off-Design Performance Characterization of a Variable Geometry Scramjet," 41st AIAA/ASME/SAE/ASEE Joint Propulsion Conference \& Exhibit, 2005, AIAA Paper 2005-3711.

[9] Bolender, M. A. and Doman, D. B., "Nonlinear Longitudinal Dynamical Model of an Air-Breathing Hypersonic Vehicle," Journal of Spacecraft and Rockets, Vol. 44, No. 2, 2007, pp. 374-387.

[10] Lind, C. A. and Lewis, M. J., "The Effect of Shock/Shock Interactions on the Design of Hypersonic Inlets," AIAA/SAE/ASME/ASEE 26th Joint Propulsion Conference, 1990.

[11] Anderson, J. D., Modern Compressible Flow with Historical Perspective, McGraw-Hill, 2002, pp. $133-142$.

[12] Smart, M. K., “Optimization of Two-Dimensional Scramjet Inlets,” Journal of Aircraft, Vol. 36, No. 2, 1999, pp. $430-433$.

[13] Torrez, S. M., Driscoll, J. F., Bolender, M. A., Oppenheimer, M. W., and Doman, D. B., "Effects of Improved Propulsion Modelling on the Flight Dynamics of Hypersonic Vehicles," AIAA Atmospheric Flight Mechanics Conference and Exhibit, 2008, AIAA Paper 2008-6386.

[14] Dalle, D. J., Torrez, S. M., Driscoll, J. F., and Bolender, M. A., "Flight Envelope Calculation of a Hypersonic Vehicle Usinga First Principles-Derived Model," 17th AIAA International Space Planes and Hypersonic Systems and Technologies Conference, 2011.

[15] Bowcutt, K., Smith, T., Kothari, A., Raghavan, V., Tarpley, C., and Livingston, J., "The Hypersonic Space and Global Transportation System: A Concept for Routine and Affordable Access to Space," 17th AIAA International Space Planes and Hypersonic Systems and Technologies Conference, 2011. 